\title{
Françoise Lalande et ses hommes tristes
}

\author{
Francisca Romeral Rosel \\ Universidad de Cádiz
}

Et revenez, toujours à la source, qui est la souffrance. (Houellebecq 1999, 11)

\section{Introduction}

Dans l'histoire de la littérature, il est des titres de romans sous lesquels pourrait être englobée dans son intégralité - ou presque - toute une œuvre. C'est le cas du titre, pour nous, révélateur de La Séduction des hommes tristes (2010); en effet, l'œuvre entière de Françoise Lalande semble se tenir sous l'égide d'une attirance, qui se verra justifiée, pour la beauté immergée dans un univers de tristesse et de survivance. Cet état d'esprit précède chez l'auteure la caractérisation des personnages de ses romans et se définit chez ces derniers en un ensemble de façons d'être au monde que l'on retrouve exposées de façon poétique chez le personnage principal, aristocrate, peintre et aventurier, de La Séduction des hommes tristes:

une révolte et une fureur l'envahissaient devant la vanité de tout destin, à moins d'être un artiste, quelqu'un qui apportait de la beauté, c'était aussi à ces heures de détresse qu'il s'interrogeait sur sa peinture, à part lui, qui connaitrait la valeur de ses tableaux?, il n'attendait plus rien des hommes, mais ce renoncement cruel s'accompagnait, il ne pouvait le nier, d'une tristesse innommable (Lalande 2010, 36).

\section{La souffrance}

S'il était possible de nommer le sujet dominant de l'œuvre de Lalande, ce serait certainement celui de la vie des malmenés de l'Histoire, des hommes - mais aussi des femmes - parmi lesquels le personnage principal de La Séduction des hommes tristes apparait comme un modèle archétypique vu qu'il concentre en grand nombre les particularités de personnages antérieurs et même postérieurs. C'est à travers ces personnageslà, et dans des histoires très différentes, que Lalande va exprimer la douleur d'être au monde, car «nommer la souffrance, l'exalter, la disséquer dans ses moindres composantes est sans doute un moyen de résorber le deuil » (Kristeva 1987, 109).

L'écrivaine y parvient tout spécialement dans le genre du roman biographique, genre qu'elle vivifie et dans lequel elle exerce une liberté à la fois ludique, audacieuse et dialectique. Cette liberté dans la recréation de l'existence de certains êtres éprouvés par le sort, bien que poétique, a le sens d'un engagement; elle n'est en effet pas dépourvue d'un positionnement 
empreint d'esprit humaniste qui se concrétise par l'écriture en une attentive considération - fondée sur le besoin de rendre justice - de l'existence singulière des "mutilés de leur société (la nôtre est-elle si différente?) » (Lalande 2012, 77). Bien que Lalande applique cette expression à Arthur Rimbaud et à Vincent Van Gogh dans la nouvelle «Ils venaient du Nord et ils étaient beaux » (2005, 75-105), elle est extrapolable à tous les personnages qui peuplent ses livres. Et ce qu'il s'agisse d'artistes réels hors du commun peintres, poètes, écrivains - fuyant toujours leur entourage étouffant, considérés comme des êtres étranges mais qui sont aussi «des seigneurs » (Lalande 2015, 96), et qui, tout comme Rimbaud et Van Gogh, ont le « corps labouré par l'orgueil et la douleur, avec cette stupeur constante de se sentir étrangers à cette planète, d'être des passants admirables » (Lalande 2005, 89); de « mystique(s) à l'état sanvage » (Claudel 1912, préface), comme l'écrivait Paul Claudel au sujet d'Arthur Rimbaud; de déclassés - aristocrates songeurs, créatifs et voyageurs - dont un exemple serait Germain Nouveau, incessamment animé par son «énergie poétique» (Lalande 2015, 96), abandonné à son déclin, fracassé de l'intérieur, être en errance parmi les habitants de son village natal où il se réfugia à la fin de ses jours; ou encore d'êtres de fiction ou de vrais marginaux sociaux ${ }^{1}$.

Tous ces êtres en souffrance nous sont présentés sous un jour nouveau, crédibles, proches et intimes, grâce à la plume de Lalande. Et ce talent s'accorde bien au rôle que l'écrivaine a joué pendant plus de cinq ans en tant qu'Administratrice d'Amnesty International Belgique. En effet, il est impossible pour Lalande de détourner le regard de la tragédie qui se joue encore ou s'est joué jadis au quotidien, comme le prouvent ses mots récents, publiés dans son souci de soutenir une famille d'exilés :

S'ils avaient le visage blême et le cœur en bouillie des émigrés en arrivant chez nous, je l'ignore. Mais je sais qu'ils nourrissaient un rêve de vie heureuse. La paix, le respect, l'instruction entre autres que mon pays leur a offerts, pourquoi veut-il aujourd'hui les en priver et renvoyer la famille Mgroyan vers un destin improbable? On n'a rien appris de l'Histoire (Lalande 2017).

Dès son premier roman Le Gardien d'abalones (1983), l'autre, enveloppé d'étrangeté, de mélancolie et d'une immense solitude, va tenir une place prépondérante dans l'œuvre lalandienne. Julia Kristeva écrit que l'un des « enjeux majeurs de la littérature et de l'art est désormais situé dans cette invisibilité de la crise qui frappe l'identité de la personne, de la morale, de la religion ou de la politique »(Kristeva 1987, 230). Déjouer l'invisibilité de l'intime, c'est justement l'enjeu que Françoise Lalande ne perd jamais de vue. De façon habile, elle se projette dans la vie et le monde de l'autre pour en saisir ce qu'il y a de plus secret, de plus délicat, d'inavouable même, et dans cette transmutation, elle produit des textes qui sont à la fois l'image de ces

\footnotetext{
${ }^{1}$ Des êtres - tels que les émigrés - auxquels Lalande prête une attention particulière dans des textes d'actualité publiés dans des revues, et qui ne feront pas l'objet de cette étude.
} 
autres et le reflet de sa propre image, dans le sens où l'appréhendait Arthur Rimbaud :

Je est un autre, écrit Arthur Rimbaud, formule qui ébranle monde comme la découverte de Galilée, elle est impérieuse comme une sentence, mystérieuse et évidente comme un proverbe, elle lacère les brouillards romantiques de la poésie, balance le poète vers une connaissance originelle, tandis que Vincent écrit de son côté je m'efforce de considérer moi-même comme un autre, car le travail de création commencera toujours par le travail sur soi-même : le poète est son propre texte, le peintre est son propre tableau (Lalande 2005, 86).

Tout comme le poète, Lalande est son propre texte; elle le transforme en un discours amoureux où l'on peut suivre la trace signifiante de son extase face à la beauté qui demeure par-delà la tristesse. Dans un court passage - un espace de trois lignes - situé à la fin de «Ils venaient du Nord et ils étaient beaux », nous pouvons constater l'intensité du sentiment amoureux qui submerge l'écrivaine à un moment précis face au spectacle offert par quelque chose de beau. Elle y raconte sa redécouverte, à l'Art Institute of Chicago, du tableau de Seurat Un dimanche après-midi à l'âle de la Grande Jatte, qu'elle avait détestée dans sa jeunesse quand elle l'avait vu reproduit dans un calendrier; cette fois, le tableau de Seurat lui donne l'opportunité de redécouvrir l'œuvre de Van Gogh. En effet, Lalande écrit au sujet de son émerveillement devant la toile de Seurat : « je fus frappée de stupeur devant le tableau, je suis tombée amoureuse de sa beauté pour toujours, il m'a dès lors renvoyée au peintre hollandais, et j'écris sur lui aujourd'hui, façon de dire l'amour» (Lalande 2005, 101). Ce sentiment amoureux, constant dans l'œuvre, est semblable à celui qu'éprouvent ses personnages, Van Gogh vivant intensément les effets de la nature qui l'entoure, comme à Arles par exemple, et Rimbaud son extase jubilatoire jusqu'à son lit de mort :

face aux strates des champs des champs moissonnés, il se mettait à trembler en effet, et c'était bien sous le coup d'une passion amoureuse et d'une colère, tornade hargneuse dont il se débarrassait comme on jouit, violence du mouvement, liberté de la vision, vertiges, et pour cela, il mettait, au bout de la main, tout le corps, comme Arthur qui rougissait sous le coup d'une émotion forte, et sans doute, parmi les émotions fortes de sa vie, il y eut la dernière à endurer, celle des heures où il achève sa vie dans une sorte de rêve continuel : il dit des choses bizarres très doucement... [...], on le croit fou, alors qu'il est entré dans le poème final, dont il se libère... (Lalande 2005, 87-88).

Comment expliquer cet intérêt pour les êtres en souffrance auréolés de beauté? Lalande n'a pas écrit d'autobiographie, même si on serait tenté de reconnaitre un alter ego de l'écrivaine dans le personnage de Lila Keil - pour ne citer que l'un des plus parlants - qui apparait dans Nous veillerons ensemble sur le sommeil des hommes. Et ce, pour plusieurs raisons, toutes en relation directe avec son histoire familiale. Tout d'abord, le matronyme, car Françoise Lalande s'appelle de son vrai nom Françoise Lalande-Keil (d'origine française par sa famille paternelle et d'origine berlinoise par sa famille maternelle, et née dans l'Ardenne belge en pleine Seconde Guerre 
mondiale ${ }^{2}$. Par ailleurs, comment ne pas voir dans le personnage de Lila Keil, petite fille de la matriarche Liza Keil - dernière représentante d'une famille poursuivie, traquée et meurtrie par la Shoah, tout comme celle de l'écrivaine ${ }^{3}$-, souffrant d'arthrose - semblablement à l'écrivaine - un double d'elle-même? Prévoyant sa transformation en "statue de pierre » (Lalande 2012, 288), Lila Keil «décide de faire une radiographie de son squelette, elle en obtient une épreuve, une photo de $1,75 \mathrm{~m}$, qu'elle affiche au mur de sa chambre, grandeur nature » (Lalande 2012, 288); elle y affichera aussi la plus connue des photos d'Arthur Rimbaud, à l'âge de seize ans, celle « retouchée par Carjat ${ }^{4}$ » (Lalande 2012, 288). Comment ne pas déceler dans l'admiration amoureuse de Lila Keil envers le poète illuminé, la même tendresse et compréhension que lui a toujours manifestée l'écrivaine? Lila Keil se réfugie dans la fiction, "méthode pour rester en vie dans un monde impersonnel » (Lieshout 2018), tout comme Lalande qui avouait lors d'une conférence : «J'ai su que mon désir de fiction se nourrissait de cette douleur secrète, secrète mais non incompréhensible $»^{5}$. Elle parle bien sûr de la douleur de se sentir autre, du besoin de partager cette douleur avec ceux qui l'endurent aussi, mais en même temps de la volonté d'être en harmonie avec ceux qui ont eu une existence tourmentée quoique non privée de beauté. Pour affronter ses maux, la jeune Lila Keil puise son énergie dans la littérature et la lutte romaine, des pratiques qui relèvent pour elle « du même domaine, celui du combat avec soi-même, celui de la descente dans les profondeurs humaines » (Lalande 2012, 149). Découvrant que le bonheur passe personnellement par l'art, elle va donner, à partir de ce moment, libre cours à son « formidable appétit de vivre » (Lalande 2012, 129).

\section{La fiction biographique}

\footnotetext{
${ }^{2}$ La Seconde Guerre mondiale a marqué fortement l'existence de Françoise Lalande. Julia Kristeva écrit au sujet de ce moment douloureux de l'Histoire : "L'actualité de la Seconde Guerre mondiale a brutalisé les consciences par l'explosion de la mort et de la folie, qu'aucun barrage, idéologique ou esthétique, ne paraissait plus pouvoir contenir. Il s'agissait d'une pression ayant trouvé au sein de la douleur psychique sa répercussion intime et inévitable. Elle fut ressentie comme une urgence inéluctable, sans pour autant cesser d'être invisible » (Kristeva 1987, 230).

${ }^{3}$ L'après-Auschwitz est une période de l'Histoire sur laquelle Françoise Lalande n'a cessé de revenir comme il en ressort des études menées par André Bénit: "Témoigner de la monstruosité de la Shoah. Le devoir de mémoire et de transmission de Vincent Engel et de Françoise Lalande» (2014) et "Françoise Lalande-Keil, mémorialiste de la Shoah en Belgique : une "méchante" histoire familiale » (2018).

4 À cette époque, la photographie la plus connue d'Arthur Rimbaud était celle réalisée en 1870 par le photographe Étienne Carjat, qui réalisa surtout des portraits de personnages importants de la fin du XIX ${ }^{\text {e }}$ siècle, tels que Paul Verlaine, Édouard Monet, Charles Baudelaire et Georges Bizet.

${ }^{5}$ Conférence «Le bonheur au bout de ma plume », prononcée dans le cadre de la Rencontre autour de l'cuvre de Françoise Lalande organisée par André Bénit (Facultad de Filosofía y Letras, Universidad Autónoma de Madrid, 24 mars 2017).
} 
En sait-on jamais assez sur les autres pour témoigner fidèlement de leur vie? La distance temporelle oblige souvent l'écrivain à imaginer, à inventer, à se tenir à l'écart quand la folie fait irruption au cours de la découverte. Cependant, Françoise Lalande se maintient à un certain degré d'objectivité quand il s'agit d'aborder la vie de personnes qui ont vraiment existé. Si l'autre est un être de fiction comme le gringo ou le gamine de La Séduction des hommes tristes, ou simplement un être réel rendu à la fiction, tel le personnage d'Alma Mahler dans la pièce de théâtre du même nom (1989), ou encore celui de la mère de Rimbaud dans Madame Rimband (1987), il est dans tous les cas issu d'un amalgame de portraits croisés, de lectures, de recherches pointues et du souvenir d'expériences bouleversantes que Lalande a vécues au cours de ses nombreux voyages - en Amérique du Sud, au Zaïre, au Maroc, entre autres -; cet autre textuel renvoie, tel un miroir, une image signifiante de l'être ayant habité le réel et, inexorablement, l'image même de l'être écrivant.

Si nous nous en tenons aux récits de Françoise Lalande dans lesquels sont abordées les vies de grandes figures de l'art et de la littérature, il en ressort qu'à l'origine de ces œuvres se trouvent la douleur et une immense passion pour la beauté. Cet alliage favorise le rapprochement entre le lecteur, attiré par l'esthétique autant du phrasé sinueux et poétique des récits fictionnels que par celle des images auxquelles ils font allusion, et séduit à la fois par les héros, qu'ils soient poètes, peintres ou aristocrates, rapprochement qui se déroule dans une ambiance d'harmonieuse proximité, d'accointance, quasi de connivence, dans laquelle l'écrivaine ne manque pas, par le biais, de faire son apparition. Qu'il s'agisse de Jean-Jacques Rousseau, d'Arthur Rimbaud, de Vincent Van Gogh ou de l'artiste plasticien Pierre Lahaut, nous entrons dans leurs alcôves ou leurs masures, nous les suivons dans leurs promenades ou leurs déboires, nous pénétrons dans leurs pensées, et nous avons même l'immense privilège de les revoir dans leur enfance. Tout est possible dans l'imaginaire littéraire de Françoise Lalande, elle voyage à travers le temps et se trouve présente auprès de ses héros : dans le recueil de nouvelles L'bomme qui aimait (2002), Rimbaud et Van Gogh ont une rencontre au «cabaret vert », « en face de la gare de Charleroi » (19-25), et, plus loin, dans «Quelque chose de bleu» (59-71), un témoin invisible assis au chevet de Rousseau assiste à l'exhalation de l'ultime souffle du grand génie contemplant sa vie; dans Pourquoi cette puissance... (2015), les derniers jours de Germain Nouveau sont évoqués par le maitre d'école du village devant un auditoire de dandys parisiens; les expérimentations langagières de Christian Dotremont et ses interventions dans le cadre du mouvement révolutionnaire Cobra sont répertoriées minutieusement par un admirateur dans Christian Dotremont l'inventeur de Cobra (1998). Cependant, la singularité de Lalande s'exprime dans son souci de se consacrer moins à une étude

\footnotetext{
${ }^{6}$ Le terme gamine désigne en Amérique du Sud l'enfant des rues, sans famille et sans loi, grandissant dans un entourage de violence et d'extrême pauvreté.
} 
critique de l'œuvre qu'à la vie de ces hommes de talent; la recréation de leur vécu, fictionnelle certainement, mais dont la composante crédible s'appuie sur des recherches rigoureuses, nous aide à mieux approcher par la suite, sous un angle inédit, leurs œuvres surprenantes.

S'il est vrai qu'il est difficile de différencier la part de vérité de la part d'invention dans les romans biographiques de Françoise Lalande, il serait toutefois possible de dire, à l'instar de Roland Barthes, que l'on y retrouve

cet appareil à la fois destructif et résurrectionnel propre à tout l'art moderne. Ce qu'il s'agit de détruire, c'est la durée, c'est-à-dire la liaison ineffable de l'existence : l'ordre, que ce soit celui du continu poétique ou celui des signes romanesques, celui de la terreur ou celui de la vraisemblance, l'ordre est un meurtre intentionnel. [...] La sincérité a ici besoin de signes faux, et évidemment faux, pour durer et pour être consommée. (Barthes 1953, 32)

Quel rapport existe-t-il entre Françoise Lalande et ces autres dont elle explore les passions, l'univers mental et le destin tragique? Comme il a été dit plus haut, le titre de l'un de ses romans, La Séduction des hommes tristes, nous semble fort éloquent : d'une part, il fait allusion à la personnalité fascinante du gringo, le héros anonyme du récit qui attire les femmes par son air malheureux, mais, de l'autre, il condense une sorte de confession de foi par laquelle l'écrivaine exprime sa propre attirance à l'égard des êtres éprouvés par les avatars de leur existence. Les hommes tristes, ce sont des « séducteurs par le mystère même de cette blessure d'amour, que les femmes devinent, attirées par la tristesse comme des abeilles butineuses, dans le défi qu'elles se lancent de guérir l'homme jusqu'à détruire le souvenir de celle qui avait planté cela dans son cœur » (Lalande 2010,31). Dans ce récit, l'homme triste, le gringo, ayant, comme une vieille épave, fini par s'échouer sur une plage du Mexique, souffre continuellement «d'une insatisfaction rapide, d'un sentiment d'ennui » (Lalande 2010, 23). Fasciné et séduit dès son jeune âge par la scène représentant l'exécution de l'empereur Maximilien dans le tableau d'Édouard Manet, par « la beauté des condamnés à mort, la chemise blanche, l'élégance des corps masculins malgré la déroute, la splendeur triste d'une histoire qui finissait mal » (Lalande 2010, 27), il va suivre sciemment ce modèle esthétique et romantique tout au long de sa vie.

\section{L'enfance}

Le vécu prend immanquablement son départ dans l'enfance, lieu de tous les possibles, dimension à la fois magique et tragique où l'on se plaît à imaginer des éternels Peter Pan qui, malgré le temps et les effroyables tempêtes de leurs existences, ne vieillissent jamais tout à fait, restent vivants d'une certaine manière. "Un poète mort n'écrit plus. D'où l'importance de rester vivant » (Houellebecq 1999, 19). Et il reste précisément vivant grâce à d'autres écrivains ou d'autres poètes qui, par leur art, parviennent à le maintenir en vie, à le faire revivre ou à le ressusciter, comme le fait Françoise Lalande. La mise en fiction de l'enfance, insérée dans le récit de vie de ces 
enfants terribles, permet à l'écrivaine d'analyser et de souligner les «destructions de l'âme » «subies dans l'enfance ou l'adolescence [...] qui vont déterminer le destin ultérieur des victimes » (Roudinesco 2017, 168). Élisabeth Roudinesco écrit à propos du psychanalyste américain Léonard Shengold, lequel, dans des travaux méritoires, s'était intéressé à ces désordres émotionnels et psychiques subis dans l'enfance et l'adolescence :

Il ne se contente pas de les décrire, il analyse comment elles déterminent le destin ultérieur des victimes. Pour survivre, le sujet préserve une image positive du parent meurtrier en justifiant ou en refoulant les mauvais traitements qui lui ont été infligés. Aussi risque-t-il ensuite de maltraiter à son tour d'autres personnes, enfants ou adultes. À travers des exemples de patients ou d'écrivains célèbres Dickens, Kipling, Tchekhov -, Shengold montre qu'il est néanmoins possible de rompre cette logique répétitive, soit par une cure psychanalytique qui conduit le sujet à dépasser son impuissance et sa révolte, soit par le développement d'un talent artistique qui le pousse vers une sublimation de son malheur (Roudinesco 2007, 168).

Françoise Lalande sait donc recueillir dans les récits de vie qu'elle nous propose ces destructions de l'âme qui ont été subies dans l'enfance, ces plaies continuellement ouvertes.

Chez le jeune Jean-Jacques Rousseau, elle met à découvert le deuil d'être orphelin de mère dès la naissance, le besoin inassouvi de combler cette absence d'amour maternel par le rêve précoce d'une vie robinsonnienne au grand air où la nature tient le rôle de mère protectrice :

Un des garçons avait appris une nouvelle étonnante : on avait trouvé un enfant sauvage du côté de Chalons. Et ces adolescents, si proches encore de l'enfance, à peine éduqués, élevés par des maittres redoutables, avaient obscurément envié l'enfant sauvage qui avait reçu de l'amour auprès d'animaux féroces. Parce que les loups n'avaient pas dévoré le bébé, mais l'avaient nourri, soigné, élevé avec le reste de la meute, les apprentis avaient ressenti de la mélancolie devant leur propre destin, si dépourvu d'affection. Pourquoi n'étaient-ils pas tous des enfants-loups? (Lalande 1993, 185)

Le jeune Jean-Jacques raconte ensuite à ses compagnons l'histoire de Romus et Romulus : «Les autres l'écoutaient, bouche ouverte. Aussi dégradés par la misère et les mauvais traitements qu'ils fussent, ils retrouvaient la grâce de leur âge et son innocence, touchés dans les profondeurs de leur cœur par l'évocation de ce destin fabuleux » (Lalande 1993, 186).

À propos de Rimbaud et de Van Gogh, enfants vagabonds ne cherchant qu’à fuir leur campagne natale, Lalande écrit: «ils ne connaissaient que les chemins de boue et de feuilles pourrissantes, cordes sur lesquelles danse l'enfant qui fugue, poings serrés au fond des poches, féroce et rieur, avide de capter l'air froid comme on savoure le bonheur de son intime solitude » (Lalande 2005, 76).

Quant à l'étranger, le gringo de La Séduction des hommes tristes, dès son jeune âge, il voulait lui aussi s'éloigner de sa famille au sein de laquelle il ne 
se sentait pas aimé, et rêvait d'une vie à la Rousseau : « quand il serait grand, il monterait vers le Nord, jusqu'à la pointe extrême du monde, il vivrait la vie modeste des gens de là-bas, et sans avoir lu Rousseau, dès l'âge de dix ans, il se concocta une vie diablement rousseauiste » (Lalande 2010, 29). Car le gringo a connu lui aussi une enfance malheureuse, privé de l'amour d'une mère lui préférant son aîné :

alors l'étranger se rappela que sa mère n'avait pas cessé de pleurer avant sa naissance, un jour de dispute entre son frère et lui, l'aîné avait informé son cadet Maman a pleuré sans arrêt pendant qu'elle t'attendait', ajoutant qu'elle n'avait pas pleuré assez vu le pauvre type qu'il était!, malgré son jeune âge, le garçonnet, blessé, avait tenté d'imaginer ce que cela représentait, une femme qui pleurait pendant neuf mois, [...] il avait entendu son oncle affirmer L'bomme que sa mère n'a pas aimé, ne sera jamais aimé des femmes [...] (Lalande 2010, 92).

Au sujet du rejet de l'enfant par la mère, Kristeva s’interroge : « Si toute femme enceinte nourrit des fantasmes de mort pour son bébé, certaines femmes ne sont-elles pas des meurtrières symboliques de leur progéniture pour sauvegarder leur propre identité narcissique? Il faudra inventer pour ces mères un nouveau complexe, le "complexe de Lady Macbeth" » (Kristeva 1985, 29). L'importance majeure du rôle tenu par la mère - signifiant-clé - dans ces désordres psychiques traverse la plupart des textes de Lalande. Il y a, en particulier, une scène terrifiante dans La Séduction des hommes tristes où une femme mexicaine, contrainte par la misère, amène son petit garçon loin du village et l'abandonne endormi au pied d'un arbre. Comme toute femme d'un milieu rural pauvre, cette femme est victime des hommes et dépend d'eux. La fureur qui naît de son impuissance la dévore; c'était une fureur qui «s'adressait au monde entier, et plus particulièrement au monde des hommes» (Lalande 2010, 99). Cet enfant abandonné deviendra par la suite un gamine, un enfant des rues, et se vengera de son destin injuste en commettant un double crime.

\section{La nature}

Le gringo, tout comme Rimbaud et Van Gogh, trouve refuge dans la nature. Il ne cesse de s'extasier devant le paysage océanique de la côte mexicaine qui chaque jour le remplit de douceur et calme son désarroi : « il aimait s'arrêter un bref instant afin de capter en un seul regard tout ce que la nature lui offrait, son cerveau photographiait en une seconde l'immensité de la plage et le Pacifique infini, du blond et du turquoise, deux espaces vastes et mouvants, nouveaux chaque soir » (Lalande 2010, 23).

Le gringo partage avec Van Gogh et Rimbaud le même amour pour la lumière. Lassé des vastes étendues glacées, il s'exile au Mexique, tandis que Van Gogh part « vers le sud à la recherche d'une lumière différente de celle du nord, il voulait voir le ciel bleu lessive du Midi, et renonça à celui de son pays, un ciel comme toujours trempé dans la mer» (Lalande 2005, 90). Quant à Rimbaud « qui s'indigne contre l'horizon fermé, la pluie et le vent 
froid, le manque de lumière », il s'en ira pour les pays chauds, «vers le soleil qui le brûlera davantage qu'il ne le réchauffera, mais au début l'homme du Nord se régale de cette chaleur nouvelle » (Lalande 2005, 92)

\section{Les amours}

Il est difficile d'aimer et d'être aimé pour ces aventuriers. Laura, la femme que le gringo aima le plus, la jeune Finlandaise blonde aux pommettes saillantes, qui «était en réalité sa sœur de tristesse, d'ailleurs, après leur rupture elle avait sombré dans la dépression » (Lalande 2010, 67), finit par le quitter parce qu'il ne veut pas partager avec elle sa tristesse, qu'il fait semblant d'être heureux : «elle avait déclaré cette chose mystérieuse Puisque c'est comme ça, je te quitte!, il n'avait pas compris pourquoi, il avait cru en mourir » (Lalande 2010, 64). Au Mexique, il accepte auprès de lui la présence d'Alegria, une jeune villageoise qu'il n'aime pas vraiment et qui ressemble par bien des aspects à l'Abyssinienne de Rimbaud. Pour le gringo, Alegria personnifie «la foule des exploités de la terre, la masse impavide en apparence, habituée à vivre de peu et à trimer, abrutie par des siècles d'oppression, colérique par soubresauts, avant de retomber dans la morne résignation des vaincus » (Lalande 2010, 45).

Quant à Rimbaud et Van Gogh, ils « eurent des amours, étranges aux autres et sans doute à eux-mêmes, de celles dont on se demande par après comment elles ont pu naître dans leur cœur » (Lalande 2005, 83). Tandis que Van Gogh tombe amoureux fou de sa cousine qui le fuit et s'éprend par la suite d'une prostituée alcoolique, Rimbaud, lui, sa relation avec Verlaine achevée, s'éprend d'une belle Abyssinienne qu'il finit par rejeter pour mettre fin à la mascarade du "colon français et sa domestique » (Lalande 2005, 84), une relation identique à celle qui s'établit entre le gringo et la jeune Mexicaine. Ces personnages, quand le voile de la tristesse se déchire, savourent enfin de courts moments de félicité : «le bonheur, bref comme un spasme, avant de renouer pour longtemps avec l'inquiétude, le doute, et même le dégoût » (Lalande 2005, 88).

\section{Conclusion}

Des êtres tels que le gringo, Van Gogh ou Rimbaud, mettent en évidence la prédilection de Lalande pour ces hommes " préférant la frugalité à l'abondance, des corps maigres pour des vies de damnés » (Lalande 2005, 79). Le gringo, personnage archétypique dans l'œuvre de l'écrivaine belge, résume à lui seul la «tristesse de l'homme qui ne serait jamais qu'un étranger, la tristesse de celui qui a compris qu'il ne serait jamais rien d'autre qu'un étranger, dans ce pays et dans tous les autres qu'il avait traversés » (Lalande 2010, 114). 
Dans des récits où se mêlent la fiction et la réalité, Lalande conjugue une remarquable intuition artistique à une recherche scientifique qui nous permet de réévaluer le parcours existentiel de grandes figures de l'art et de la littérature, de certains de leurs proches ou de personnages de fiction, toujours à travers le sentiment aigu de la douleur et le sens de la beauté. Pour se faire une idée visuelle de cette démarche, on peut utiliser l'un des rêves d'Akira Kurosawa ${ }^{7}$, Les corbeaux, court-métrage aux couleurs éclatantes, dans lequel le réalisateur japonais met en images sa passion pour la peinture de Van Gogh, à travers la figure d'un Van Gogh halluciné - interprété par Martin Scorsese - par la beauté d'un champ de blé sous un soleil éblouissant. Lalande traduit en une phraséologie poétique ce regard explicatif tourné à la fois vers l'extérieur et vers elle-même qui nous introduit dans les coulisses de l'art entrevu comme mécanisme de défense face aux obstacles de la vie. Ce talent créateur, comme nous l'avons vu chez Rimbaud, Van Gogh, Rousseau et l'étranger de La Séduction des hommes tristes, ne suffit pas, la plupart du temps, à calmer les conflits internes, et risque même de les exacerber. Cependant, cette faiblesse d'origine psychique n'altère jamais la séduction pour la beauté dans la tristesse.

\footnotetext{
${ }^{7}$ Le film Les rêves de Akira Kurosawa, sorti en 1990, est une série de huit court-métrages réalisée par Akira Kurosawa et Ishirō Honda. Il est composé de huit rêves que fit Kurosawa lui-même et dont il écrivit le scénario et fit des dessins préparatifs.
} 


\section{Bibliographie}

Barthes, Roland. Le Degré zéro de l'écriture. Paris : Seuil, 1953.

Bénit, André. "Témoigner la monstruosité de la Shoah. Le devoir de mémoire et de transmission de Vincent Engel et Françoise Lalande ». Les monstres. Éd. Catherine Gravet \& Pierre Gillis. Cabiers internationaux de symbolisme, n'137, 138-139, 2014 : 37-54.

---. «Françoise Lalande-Keil, mémorialiste de la shoah en Belgique : une "méchante" histoire familiale ". Guerra y violencia en la literatura y en la bistoria. Éd. Fernando Carmona Fernández y José Miguel García Cano. Universidad de Murcia / Museo de la Universidad de Murcia / Centro de Estudios del Próximo Oriente y la Antigüedad Tardía, $2018: 117-126$.

Claudel, Paul. «Préface » aux CEures d'Arthur Rimbaud, repris dans Positions et propositions. $\quad$ Paris : 1928. http://www.biblisem.net/etudes/claudrim.htm (consulté le 29/01/2018).

Houellebecq, Michel. Rester vivant. Paris : Flammarion, 1999.

Kristeva, Julia. Au commencement était l'amour. Psychanalyse et foi. Paris : Hachette, 1985.

---. Soleil Noir. Dépression et mélancolie. Paris : Gallimard, 1987.

Lalande, Françoise. Le Gardien d'abalones. Bruxelles: Éditions Jacques Antoine, Coll. Écrits du Nord, 1983.

---. Madame Rimbaud. Paris : Presses de la Renaissance, 1987.

---. Alma Mabler. Paris : Actes Sud -Papiers, 1989.

---. Jean-Jacques et le plaisir. Paris : Belfond, 1993.

---. Christian Dotremont, l'inventeur de Cobra. Une biographie. Paris : Stock, 1998.

---. L'Homme qui aimait. Bruxelles : Éditions Le Grand Miroir, Coll. La petite littérature, 2002.

---. " Ils venaient du Nord et ils étaient beaux ». Dans les replis nocturnes de mon cour. Bruxelles : Editions Luc Pire \& Le Grand Miroir, 2005, 75-105.

---. La Séduction des hommes tristes. Avin : Éditions Luce Wilquin, 2010.

---. Pierre Lahaut. Portrait et autoportraits. Repères biographiques. Bruxelles : Éditions Fonds Mercator, 2011.

---. Nous veillerons ensemble sur le sommeil des hommes. Avin : Éditions Luce Wilquin, 2012.

---. Pourquoi cette puissance... Germain Nouveau. Avin : Editions Luce Wilquin, 2015.

---. «Les trois phrases de Françoise Lalande». https://soutenirlafamillemgroyan.wordpress.com/2017/03/03/lestrois-phrases de-francoise-lalande/(consulté le 18 novembre 2017).

Lieshout, Erik et Van Brummelen, Reinier. Rester vivant: méthode. Film (Damned Distribution, 2018). D'après l'essai de Michel Houellebecq du même titre (Paris: Flammarion, 1999) https://www.franceculture.fr/evenement/rester-vivant 
methode-derik-lieshout\#xtor=EPR-2-\%5BLaLettre10052018\%5D (consulté le 9 mai 2018).

Roudinesco, Élisabeth. Dictionnaire amoureux de la psychanalyse. Paris: Plon/Seuil, 2017. 